\title{
Control of Non-Linear Inverted Pendulum using Fuzzy Logic Controller
}

\author{
Arpit Jain \\ Deep Tayal \\ Neha Sehgal \\ University of Petroleum \& Energy Studies, Dept of Electrical, Electronics \& Instrumentation Engineering \\ Dehradun, India
}

\begin{abstract}
This paper proposes an intelligent control approach towards Inverted Pendulum in mechanical engineering. Inverted Pendulum is a well known topic in process control and offering a diverse range of research in the area of the mechanical and control engineering. Fuzzy controller is an intelligent controller based on the model of fuzzy logic i.e. it does not require accurate mathematical modelling of the system nor complex computations and it can handle complex and non linear systems without linearization. Our objective is to implement a Fuzzy based controller and demonstrate its application to Inverted Pendulum. Model design and simulation are done in MATLAB SIMULINK ${ }^{\circledR}$ software.
\end{abstract}

Keywords-Inverted Pendulum, Fuzzy logic, Fuzzy controller

\section{INTRODUCTION}

The fact that fuzzy systems are universal approximators has been shown and proven by several sources [1, 2]. These proofs arise from the isomorphism between two algebras: an abstract algebra (one dealing with groups, fields, and rings) and a linear algebra (one dealing with vector spaces, state vectors, and transition matrices) and the structure of a fuzzy system which constitutes of an implication between actions and conclusions. This isomorphism is achieved because both entities involve a mapping between elements of two more domains. Like an algebraic function maps an input variable to an output variable, a fuzzy system maps an input group to an output group: in the latter these group can be linguistic assertions or forms of fuzzy information. The underlying principle on which fuzzy systems theory rests is a fundamental theorem from real analysis in algebra known as Stone - Weierstrass theorem, first developed in the late nineteenth century by Weierstrass[3], then simplified by Stone[4].

Although fuzzy systems are shown to be universal approximators to algebraic functions, it is not this attribute that actually makes them valuable to us in grasping new or evolving problems. Rather, the primary advantage of fuzzy systems theory is to approximate system behaviour where analytic functions or numerical relations are absent. Therefore, fuzzy systems have high potential to understand pivot point in a feedback system is achieved by balancing an upturned broomstick on the end of one's finger. The inverted pendulum is a standard problem in dynamics and control the very systems that lack analytic formulations: complex systems.

Instead, fuzzy systems theory can be useful in assessing some of our more conventional, less complex systems. For example, suppose a controller is required to bring an aircraft out of a vertical dive. Conventional controllers, as they are restricted to linear ranges of variables, cannot handle this scenario; a dive situation is highly non-linear. Fuzzy controller can be used in this case, which is adept at handling non linear situation albeit in an imprecise fashion, to bring the plane out of the dive into a more linear range, then handoff the control to the aircraft to a conventional, linear, highly precise controller.

As pointed out by Ben - Haim[5], this is a distinction between models of systems and models of uncertainty. A fuzzy system can be thought of as a collection of both because it attempts to understand a system for which no model exist, and it does so with information that can be uncertain in a sense of being vague, or fuzzy, or altogether lacking. Fuzzy systems are robust because the uncertainties contained in both the inputs and outputs of the system are used in formulating the system structure itself, unlike conventional system analysis which first poses a model, based on a collective set of assumptions needed to formulate a mathematical form, then uncertainties in each of the parameters of that mathematical abstractions are considered which could be misleading.

\section{INVERTED PENDULUM ON A CART}

A pendulum which has its center of mass above its pivot point is known as an Inverted Pendulum. It is usually implemented with the pivot point mounted on a cart that can move horizontally and may be called a cart and pole. Many applications limit the pendulum to 1 (single) degree of freedom by affixing the pole to an axis of rotation. While a normal pendulum is stable when hanging downwards, an inverted pendulum is inherently unstable, and must be aptly balanced in order to remain upright; this hasbeen done either by application of a torque at the pivot point, by moving the pivot point in horizontal motion as part of a feedback system, altering the rate of rotation of a mass mounted on the pendulum on an axis parallel to the pivot axis and thereby generating a net torque on the pendulum, or by oscillation of the pivot point vertically. A simple demonstration of moving the

theory and is used as a benchmark for testing control strategies. 
The inverted pendulum is among the most difficult systems being an inherently unstable system, is a common control problem, and so being one of the most significant classical problems, the control of inverted pendulum has been a research interest in the field of control engineering. Due to its importance this is a choice of dynamic system to analyze its dynamic model. The aim of this case study is to stabilize the Inverted Pendulum (IP) such that the position of the cart on the track is controlled rapidly and accurately so that the pendulum is always in erected in its inverted position during such movements even the system is affected with disturbance input such as wind force or due to others cause. Realistically, this mechanical system is representative of a class of altitude control problems whose aim is to maintain the desired vertically oriented position at all times [6-9].

In general, the control problem consists of obtaining dynamic models of systems, and using these models to determine control laws or strategies to achieve the desired system response and performance. Keeping the control algorithm simple as well as guaranteeing the stability and robustness in the closed-loop system is a challenging task in real situations. Most of the dynamical systems such as power systems, robotic systems, inverted pendulum, industrial processes, chaotic circuits etc. are highly nonlinear in nature. The control of such systems is a challenging task.

Development in the area of artificial intelligence (AI), such as artificial neural network (ANN), fuzzy logic theory (FL), and evolutionary computational techniques such as genetic algorithm (GA), and particle swarm optimization (PSO) etc., commonly all these are known as intelligent computational techniques[10-12]. Intelligent control is a feasible recent approach which has been emerged from the integration of control methodologies with intelligent computational techniques.

Due to the simple approach fuzzy control provides the use of heuristic control knowledge for nonlinear control problems. The past few years have witnessed a speedy growth in the number and various applications of fuzzy logic [13-19].

\section{a. MATHEMATICAL MODELLING}

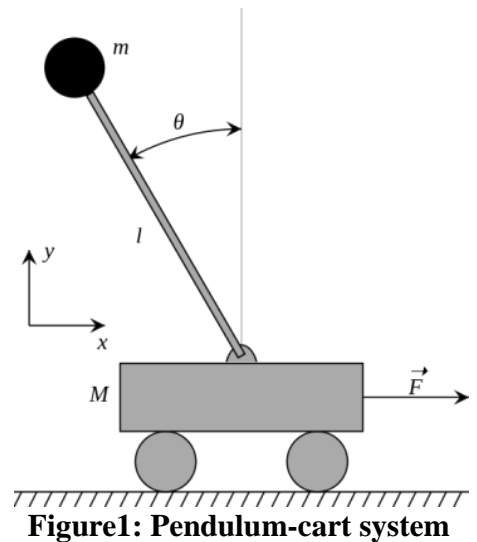

$M=$ mass of cart

$\mathrm{m}=$ mass of pendulum

$1=$ length of rod

$\mathrm{g}=$ acceleration due to gravity
An inverted pendulum on a cart consists of having a horizontally moving base as shown in the image to the right. The cart is restricted to linear motion and is subjected to forces resulting in or hindering motion. The equations of motion can be derived using Lagrange's equations. We refer to the drawing to the right where $\theta(t)$ is the angle of the pendulum of length 1 with respect to the vertical direction and the acting forces are gravity and an external force $F$ in the xdirection. Define $\mathrm{x}(\mathrm{t})$ to be the position of the cart.

$$
\begin{gathered}
(M+m) x^{\bullet \bullet}-m l \theta^{\bullet \bullet} \cos \theta+m l \theta^{\bullet 2} \sin \theta=F \\
l \theta^{\bullet \bullet}-g \sin \theta=x^{\bullet \bullet} \cos \theta[20-21]
\end{gathered}
$$

State Space Analysis:

$$
\begin{gathered}
X^{\bullet}=A x+B y \\
Y^{\bullet}=C x+D y \\
{\left[\begin{array}{l}
X_{1} \\
X_{2} \\
X_{3} \\
X_{4}
\end{array}\right]=\left[\begin{array}{cccc}
0 & 0 & 1 & 0 \\
0 & 0 & 0 & 1 \\
0 & -\alpha & 0 & 0 \\
0 & \gamma & 0 & 0
\end{array}\right]\left[\begin{array}{l}
x_{1} \\
x_{2} \\
x_{3} \\
x_{4}
\end{array}\right]+\left[\begin{array}{c}
0 \\
0 \\
\beta l \\
-\beta
\end{array}\right] \mathrm{u}} \\
{\left[\begin{array}{l}
Y_{1} \\
Y_{2}
\end{array}\right]=\left[\begin{array}{cccc}
1 & 0 & 0 & 0 \\
0 & 1 & 0 & 0
\end{array}\right]\left[\begin{array}{c}
x_{1} \\
x_{2} \\
x_{3} \\
x_{4}
\end{array}\right]} \\
\mathrm{Y}=\left[\begin{array}{llll}
0 & 1 & 0 & 0
\end{array}\right]\left(\begin{array}{c}
x_{1} \\
\vdots \\
x_{n}
\end{array}\right) \\
\alpha=\frac{g}{l(M+m)-1} ; \quad \beta=\frac{1}{l(M+m)-1} \\
\gamma=\frac{(M+m) g}{l(M+m)-1}[22]
\end{gathered}
$$

These equations are nonlinear, but since the goal of a control system would be to keep the pendulum upright the equations can be linearized around $\theta=0$.

\section{i. PARAMETERS}

The table below shows the parameters that have been taken during the study.

TABLE 1

\begin{tabular}{|l|l|}
\hline $\mathrm{M}$ & $0.5 \mathrm{Kg}$ \\
\hline $\mathrm{M}$ & $0.2 \mathrm{Kg}$ \\
\hline $\mathrm{L}$ & $0.3 \mathrm{~m}$ \\
\hline $\mathrm{G}$ & $9.8 \mathrm{~m} / \mathrm{s}^{\wedge} 2$ \\
\hline
\end{tabular}

\section{FUZZY LOGIC CONTROLLER}

Fuzzy Logic Controller is one of the utmost thriving applications of fuzzy set theory. This control technique banks upon the human bility to understand the system's behaviour and it comprises of a set of qualitative control laws.[23] 


\subsection{Membership Functions}

Primarily, 3 membership functions have been made use of, namely:

$$
\begin{gathered}
\vartheta=\text { Pendulum Angle } \\
\theta^{\bullet}=\text { Angular Velocity } \\
F=\text { Force }
\end{gathered}
$$

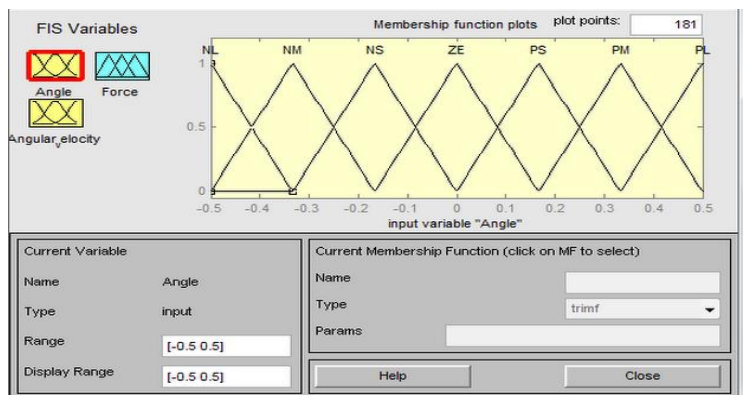

Figure2: Membership Function of Pendulum Angle

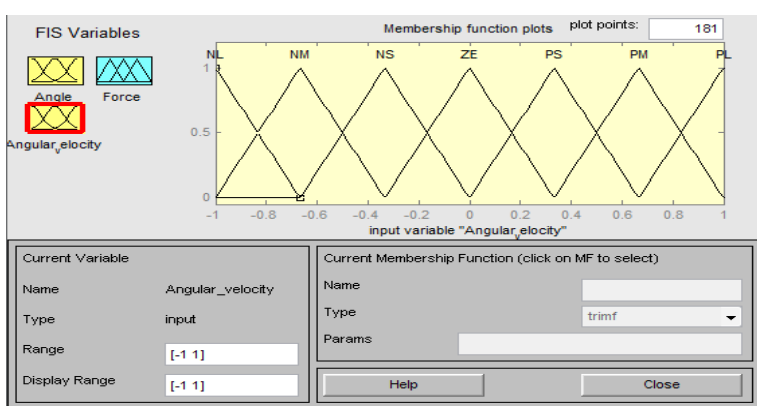

Figure 3: Membership Function of Angular Velocity

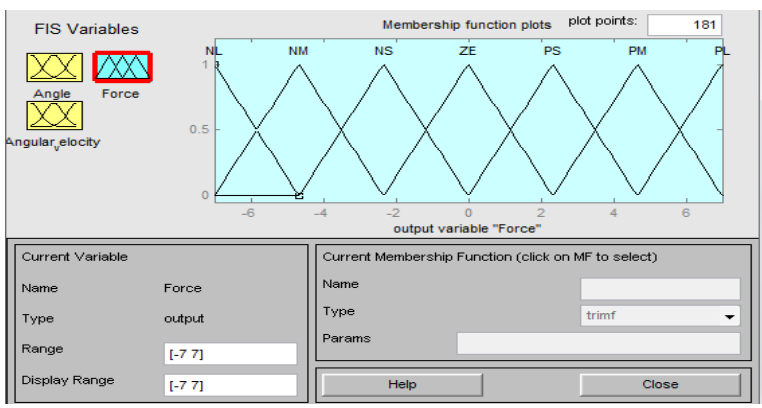

Figure 4: Membership Function of Force

Rule Base for pendulum system is shown below:

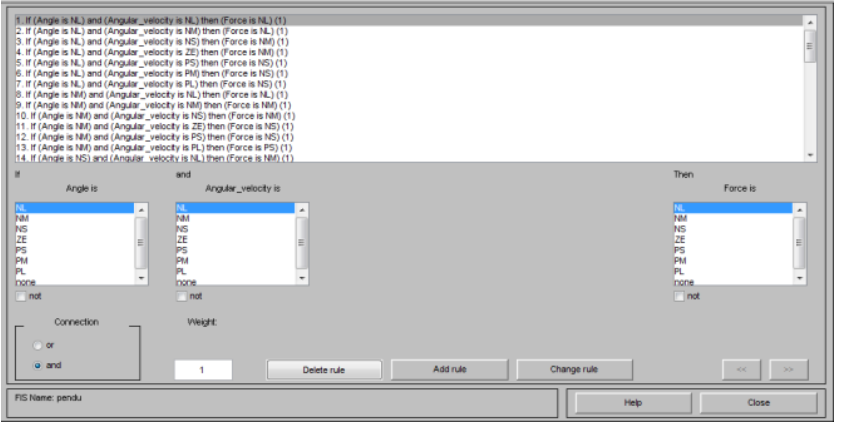

Figure5: Rule base for Pendulum Angle using Mamdani Type Fuzzy Inference System

\section{RESULTS AND CONCLUSION}

In this paper, application of Fuzzy controller to inverted pendulum has been presented. The main issue that arises while applying the Fuzzy model to an application is defining the rule base in such a way so as to approximately represent the state and objective of the system.

The model was tested on Inverted Pendulum in the presence and absence of noise. Formulations are made in the following way:

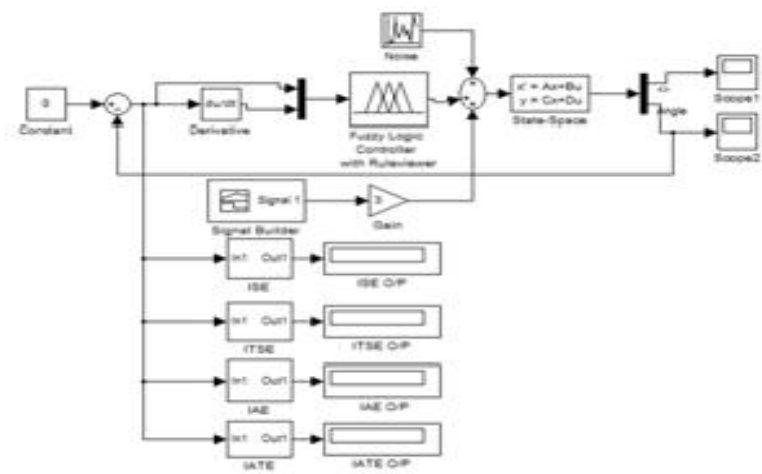

Figure 6: Fuzzy System with noise

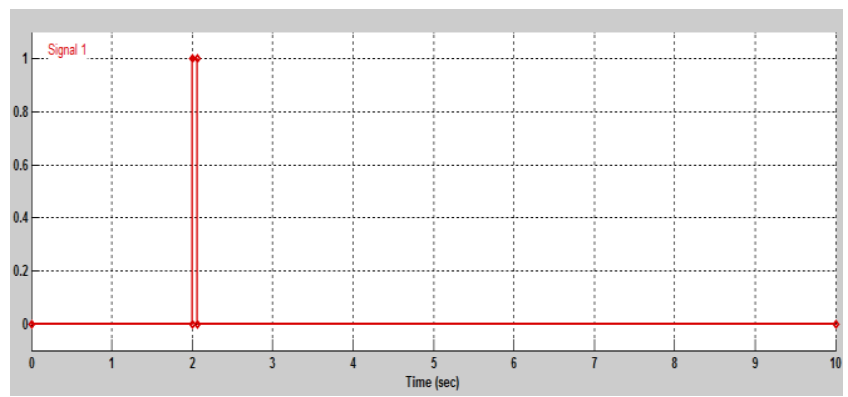

Figure 7: Single pulse disturbance input 


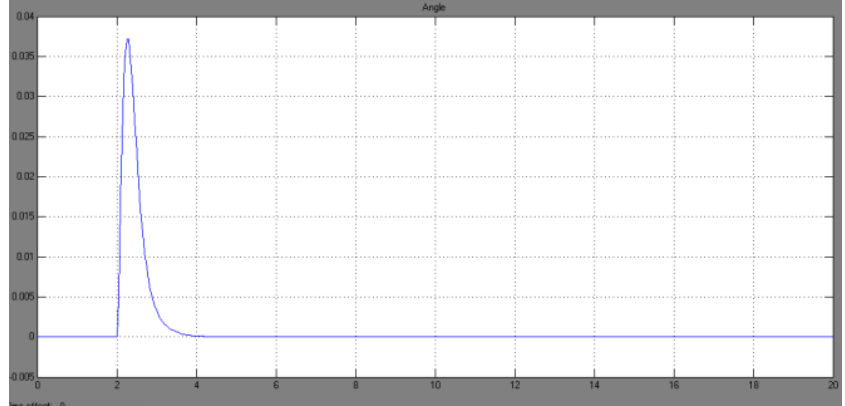

Figure 8: Output Response with single impulse

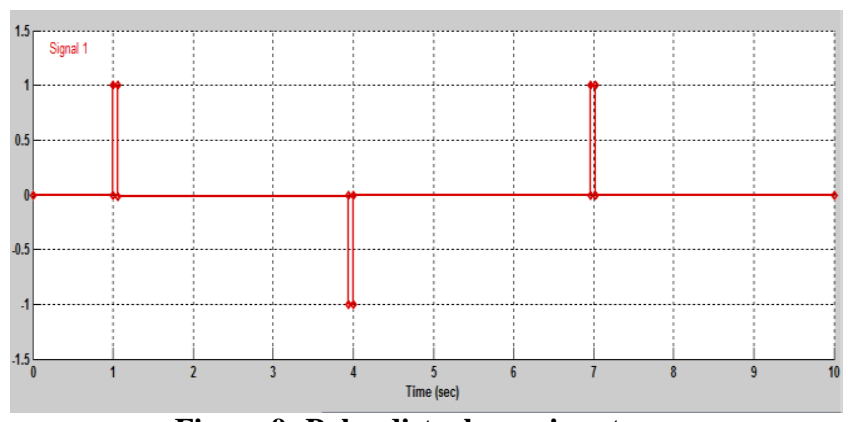

Figure 9: Pulse disturbance input

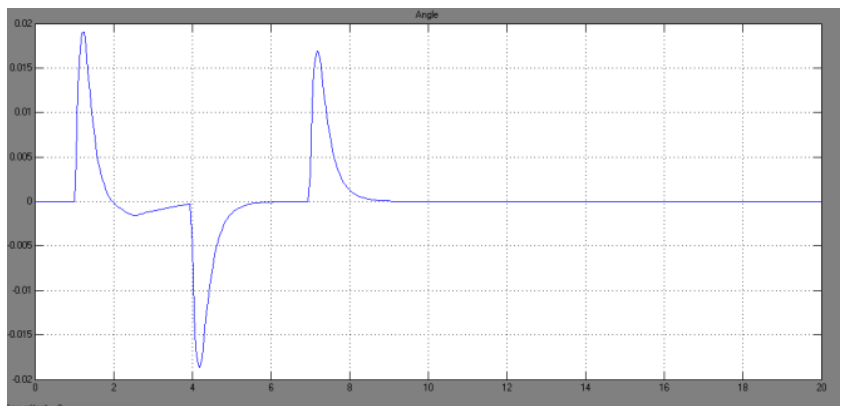

Figure 10: Output Response of System in absence of noise

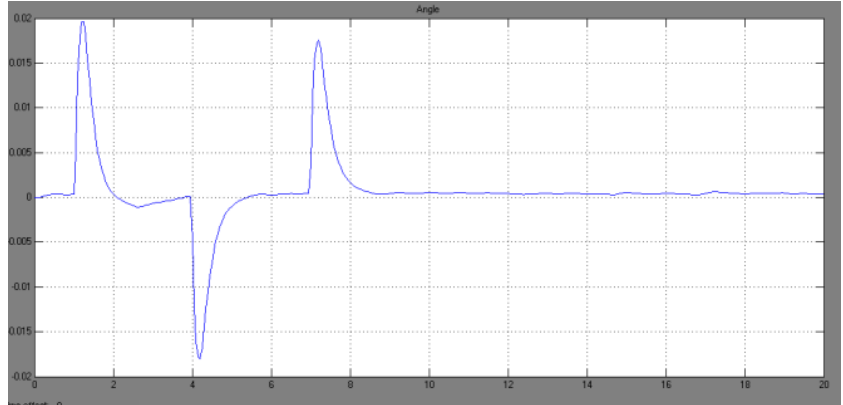

Figure 11: Output Response of System in presence of noise

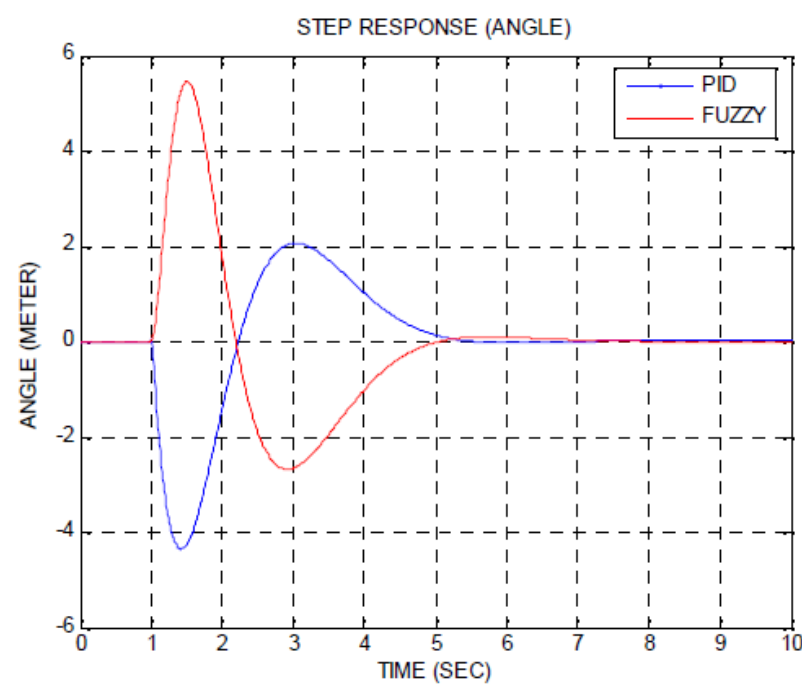

Figure 12: Pendulum's Angle Response of reference paper[23]

The above figure (figure 9) represents a pulse disturbance input, to which impulses have been applied, first in the positive direction, then in the negative and again in the positive direction. Theses impulses have been deliberately applied to the pendulum in order to check the stability, erectness and validation of performance indices of the pendulum.

Figure 6 affirms the output response of the fuzzy based inverted pendulum system which can be comprehended by viewing that initially when impulses were applied, both in the positive and negative direction, the pendulum retains its steady state position within a small lapse in time and proves the detergency of the fuzzy logic controller.

\subsection{Performance Indices}

TABLE 2

\begin{tabular}{|l|l|l|}
\hline & $\begin{array}{l}\text { Without } \\
\text { Noise }\end{array}$ & With Noise \\
\hline ISE & 0.0003186 & 0.000328 \\
\hline ITSE & 0.001318 & 0.001381 \\
\hline IAE & 0.02756 & 0.03279 \\
\hline IATE & 0.1193 & 0.1971 \\
\hline
\end{tabular}

The paper essentially encompasses in it the basics and work on an intelligent controller named "Fuzzy Logic Controller". It primarily includes the study of fuzzy logic and then its application on non linear Inverted Pendulum. Predominantly, corroboration on the steadiness, erectness and validation of the performance indices for an inverted pendulum has been performed, both in the presence and absence of noise. The results drawn have been shown in Figure 6 and in Table 2 . 
The simulation results indicate the relative vantages of fuzzy control methods. The pendulum stabilizes in upright position with bankable oscillations. The analysis of the system with the proposed control scheme gives the error parameter values: ISE $=0.0003186, \mathrm{ITSE}=0.001318, \mathrm{IAE}=0.02756$ and IATE $=$ 0.1193 . The settling time for the system with proposed controller comes out to be 4.9 seconds, which has been ascertained with a pronounced research paper [23], with their value of settling time being 6.2 seconds

\section{REFERENCES}

[1] Kosko, B. (1994). "Fuzzy systems as universal approximators," IEEE Trans. Comput, vol. 43, no. 11.

[2] Ying, H., Ding, Y., Li, S., and Shao, S. (1999). "Fuzzy systems as universal approximators," IEEE Trans. Syst., man, Cybern - Part A: Syst. Hum., vol 29, no. 5.

[3] Weiestrass, K. (1885). " mathematische Werke, Band 3, Abhandlungen III," pp. 1 - 37, esp. p. 5, Sitzunsber. Koniglichen preuss. Akad. Wiss., July 9 and July 30.

[4] Stone, M. H. (1937). "Applications of the theory of Boolean rings to general topology," Trans. Am. Math. Soc., vol. 41, pp. 375 - 481, esp. pp. 453 - 481.

[5] Ben-Haim, Y. (2001). Information Gap Decision Theory: Decisions Under Severe Uncertainty, Series on Decision and Risk, Academic Press, London.

[6] K. Ogata, Modern Control Engineering, $4^{\text {th }}$ ed, Pearson Education (Singapore) Pvt. Ltd., New Delhi, 2005, ch. 12.

[7] K. Ogata, System Dynamics, $4^{\text {th }}$ ed, Pearson Education (Singapore) Pvt. Ltd., New Delhi, 2004.

[8] J. R. White, System Dynamics: Introduction to Design and Simulation of Controlled Systems, Online literature.

[9] Ajit K. Mandal, Introduction to Control Engineering, New Age International Pub., New Delhi, 2000, ch. 13.

[10] Roland S. Burns, Advanced Control Engineering, Elsevier - Butterworth Heinemann, 2001, ch. 10.

[11] Astrom K. J., and McAvoy Thomas J., "Intelligent Control", J. Proc. Cont. 1992, Vol2, no 3, pp 115 - 127.

[12] T. I. Liu, E. J. Ko, and J. Lee, "Intelligent Control of Dynamic Systems", Journal of the Franklin Institute, Vol. 330, No. 3, pp. 491 - 503, 1993.

[13] Kevin M. Passino, and Stephen Yurkovich, Fuzzy Control, Addison Wesley longman, Inc., California, 1998.
[14] M. A. Denai, F. Palis, and A. Zeghbib, "Modelling and control of non-linear systems using soft computing techniques", Elsevier Journal of Applied Soft Computing, vol. 7, 2007, pp $728-738$.

[15] Lal Bahadur Prasad, Krishna Pratap Singh, and Hema Latha Javvaji, "Simulation of Neuro-Fuzzy Position Controller for Induction Motor Drive using Simulink", Proceedings of XXXI National Systems Conference, NSC 2007, P-49, Dec. 14 - 15, 2007, MIT Manipal, India.

[16] G. Ray, S. K. Das, and B. Tyagi, "stabilization of Inverted Pendulum via Fuzzy Control”, IE(I) Journal-EL, vol. 88 , Sept. 2007, pp. $58-62$.

[17] C. W. Tao, J. S. Taur, C. M. Wang, and U. S. Chen, "Fuzzy hierarchical swing-up and sliding position controller for the inverted pendulum-cart system", Elsevier Journal: Fuzzy Sets and Systems, vol. 159, 2008, pp. $2763-2784$.

[18] Yanmei Liu, Zhen Chen, Dingyun Xue, and Xinhe Xu, "Real-Time Controlling of Inverted Pendulum by Fuzzy Logic", Proceedings of the IEEE International Conference on Automation and Logistics, Shenyang, China, August 2009, pp. $1180-1183$.

[19] Hari Om Gupta, Lal Bahadur Prasad and Barjeev Tyagi, "Intelligent Control of Non Linear Inverted Pendulum Dynamical System with Disturbance Input Using Fuzzy Logic Systems", International Conference On Recent Advancements in Electrical Electronics and Control Engineering, 2011.

[20] Ashab Mishra, and Capt. Dr. Sarfraz Hussain, "Robust Controller for Nonlinear and Unstable system: Inverted Pendulum”, ASME Journal of Control \& Design Simulation, pp 49-60, vol. 55, No. 3, 4. 2000.

[21] Ashab Mishra, Iram Mahboob and Capt. Dr. Sarfraz Hussain, "Flexible Broom Balancing". ASME Journal of C \& D Simulation, vol 56, No 1, 2. 2001.

[22] Raymond T. Stefani, Bahram Shahian, Late Clement J. Savant, and Late Gene Hosteller, "Design of Feedback Control Systems", Oxford University Press, 2002.

[23] Aleem Ahmad Khan, and kashan Hussain, "Comparative Performance Analysis between Fuzzy Logic Controller (FLC) and PID Controller for an Inverted Pendulum", International Journal of Electrical, Electronics and Computer Systems (IJEECS), Vol 10, October 2012. 